\title{
Monetizing Evaluation Model for Highway Transportation Rules Based on CA
}

\author{
Yiling Liu ${ }^{1, *}$, Jin Xiong ${ }^{2}$, Xingyue Han $^{3}$ and Hong Duan ${ }^{1}$ \\ ${ }^{1}$ College of Information System and Management, National University of Defense Technology, Changsha, Hunan, China \\ ${ }^{2}$ College of Mechatronic Engineering and Automation, National University of Defense Technology, Changsha, Hunan, China \\ *Corresponding author
}

\begin{abstract}
Most of the traffic rules are originated in some historical reasons; it is difficult to say which is better than others. This paper proposed a monetizing evaluation model for highway transportation, which is based on the cellular automation (CA), more specifically, the NaSch model. We evaluated the Keep-Right rule, as an example, with the proposed model, and proposed some new rules according to the results. The fact that these rules are actually promoted in most countries proved that the evaluation model we proposed is objective and effective.
\end{abstract}

Keywords-transportation simulation; CA; traffic rule evaluation; monetizing model

\section{INTRODUCTION}

There are lots of rules for road transportation all over the word; and the rules vary from country to country. For instance, some countries, like China and the US, asked drivers to drive on the right lane except passing others, while the British Commonwealth countries and some former British colonies carrying out the Keep-Left-Except-to-Pass rule. It is reported that both of the two regulations are originated in some historical reasons. It is necessary to construct an evaluation model to help decision makers to judge whether a traffic rule is "better" than another similar one, offering them scientific evidences.

First of all, we should find out how to present a performance of a traffic rule. Intuitively, we regard a traffic rule as a good one firstly because it ensures security under it. What's more, a "better" traffic rule must promote better traffic flow. But we know these two performances are constraint each other. To balance the two indexes, we choose the monetize model [1] quantify the performance of a traffic rule: traffic flow could make profits while insecurity might bring cost.

Most of the traffic low models are solved by the Cellular Automaton (CA). Von Nenmann was the first person to advance the model [2], and Cremer and Ludwim firstly used CA to describe traffic flow models [3]. Zheng Rongsen and his team built a multi-speed traffic flow model based on CA, and analyzed different factors that impact on traffic flow apparently [4], which accords with some realities in highway but assumed that there exists no circumstance of passing. Zhu Changsheng did some research on effect of lane changing probability on traffic flow, aiming at the lane problem in the cross intersection of two-dimensional traffic network with traffic light [5]. Wang Yongming discussed safe space for lane-changing and solved the modified model [6], and put forwarded a rule about flexible safe space. They all have something focused on but mostly ignored the uncertainty brought by human. We introduced some random variables to describe people's behaviors and also considered the probability for people to pass vehicles in front of them.

The remaining of this paper is structured as follows: Section II introduced the monetize model with the two aspects we discuss. Section III describes the CA model to simulate traffic flow considering human behavior. Section IV evaluates the Keep-Right-Except-to-Pass rule using our model, as an example. Section $\mathrm{V}$ gives out some concludes and proposes some further research issues.

\section{The MONETIZE MODEL}

The monetize model, as the words imply, aims at quantifying the performance of a traffic rule by measuring profits and costs that it causes.

\section{A. Indexes}

We have some essential indexes in the monetizing model that need to clarify before build the model:

\section{1) Safety index}

Considering that only when a car is changing lane it will take some risks, we defined danger efficiency $(\varepsilon)$ for cars that are changing lanes:

$$
\varepsilon=k \cdot \frac{v}{d}
$$

Among them, $v$ is the relative speed between the car and the one behind it on the new lane, while $d$ is the relative distance. We chose them to define our danger efficiency for most of accidents happened on the highway are pileups, causing by inappropriate lane-changing (conclusion from data on the website of the USA Traffic Bureau).

Then we sum up all the $\varepsilon$ we have got, as one of the basis to calculate monetize results, and record the result as $f$.

\section{2) Traffic flow index}

Traffic flow $(q)$ is given by:

$$
q=\frac{N_{1}}{t}
$$


where $N_{1}$ is the number of cars passing the section in lasting for a period of $t$.

\section{B. The Complex Monetizing model}

We define the monetize formula as followed, referring to the reference [7]:

$$
W(q, f)=\frac{k}{q}+a f^{b}
$$

Among them, $q$ and $f$ are the indexes mentioned before, and could get with the simulation results. $k, a$ and $b$ are 3 undetermined coefficients. And $W(q, f)$ presents the "cost" on the road under the certain circumstance. Since the two indexes are related with each other and equally important, there is a total principle when determine the three coefficients:

- $\quad$ They should cause a same variety of $W(q, f)$ when varied respectively.

So we have the principle in mathematical form as :

$$
W(q+\delta, f)=W(q, f+c \delta)
$$

Then we can get all of the undetermined coefficients from our simulation results.

\section{Simulation Model BASED ON CA}

As we chose cellular automaton to simulate the traffic flow, each part of the cellular automaton should be defined.

We regard vehicles on the highway as cells in CA, and define speed and position as status set. And we know that the cells are all in the same as we assumed before. So it is obviously that the model is two-dimensional, where every vehicle could move vertically or horizontally.

The following flow diagram displays the whole process of our simulation model.

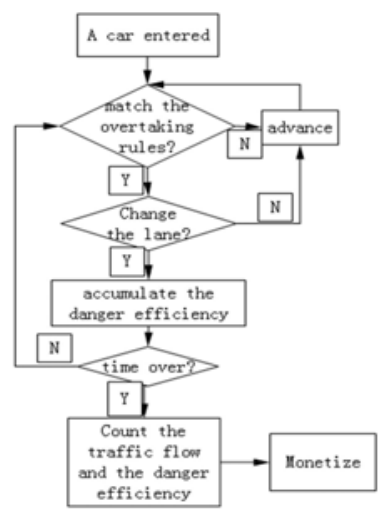

FIGURE I. A FLOW DIAGRAM FOR THE WHOLE SIMULATION PROCESS

\section{A. Model for Vehicles}

We abstract cars into cells for CA, and have the complete description of our CA model as followed:
Discretize lanes of highway to form the two-dimensional cellular space $C^{2}$, where $x_{i j}$ is one of the cells and stands for the $j^{\text {th }}$ vehicle on the $i^{\text {th }}$ lane. The probable status set is $D\left(x_{i j}\right)=\{v(i, j), s(i, j)\}$. The status $v(i, j)$ for a cellular stands for the speed of the vehicle at present, while $s(i, j)$ stands for the position in the cellular space.

Based on the NaSch model ${ }^{[8]}$, we have the evolution laws as followed:

We have a posted maximum speed and a minimum speed for the highway, and record them as $V_{\max }$ and $V_{\min }$. Set a matrix $G=\{\operatorname{gap}(i, j)\}$ record the distance between the cellular and the one in front of it, which is determined by cellular neighborhood. That is to say:

$$
\operatorname{gap}(i, j)=s(i, j-1)-s(i, j)
$$

Consider a dell in state of $D^{t}\left(x_{i j}\right)$ :

- Rule for acceleration:

$v^{t+1}(i, j)=v^{t}(i, j)+1$ when $v^{t}(i, j)<V_{\max }$.

- Randomization:

$v^{t+1}(i, j)=\max \left\{V_{\min }, v^{t}(i, j)-1\right\}$ with a certain chance $p_{1}$.

- Rule for deceleration:

$v^{t+1}(i, j)=\max \left\{\operatorname{gap}(i, j), V_{\min }\right\} \quad$ when $\operatorname{gap}(i, j)<v^{t}(i, j)$ with a chance of $p_{2}\left(p_{2}\right.$ is given in section $\left.C\right)$.

- Car-appearing coefficient:

The car-appearing coefficient ( $\alpha$ ) means that there will be some cars at the entrance of the studying section with a chance of $\alpha$.

\section{B. Lane-Changing Model}

We have FIGURE II telling relationships among a cell and it's neighborhood:

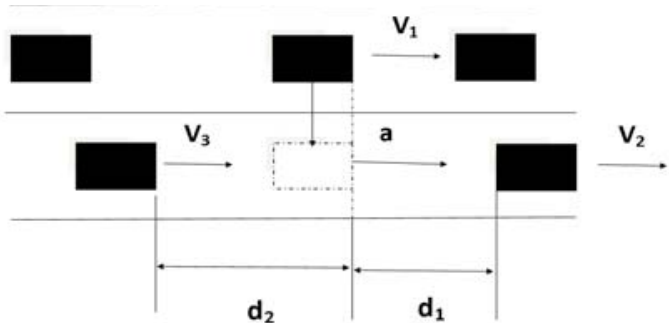

FIGURE II. RELATIONSHIPS AMONG A CELL AND IT'S NEIGHBORHOODS

There are 3 prerequisites for lane-changing:

- There is no car on the near lane: 
$s(i \pm 1, j)=0$, whether it take "+" or "“." is determined by whether the car is going to pass the former one by left or right.

- Meet the distance condition with car in front of it:

$\operatorname{gap}(i, j)>$ min_Dis, where min_Dis is the minimum distance between two cars driving on the road according to the traffic rules.

- The driver believes that he will be safe when changing the lane:

That means $d_{1} \geq v_{2}$ in FIGURE II.

A car could change lane once satisfied these prerequisites.

\section{Human Behavior}

Human behavior could bring much uncertainty to the CA model. Here we analyze some important impacts and try to quantize them by some probability coefficients or random variables.

- Probability for lane-changing

When a car meets the three prerequisites we mentioned before, the driver might decide to drive ahead or change the lane. We assume that the driver might change the lane with a chance of $p_{2}$, which could express the behavior of drivers when the car meets the prerequisites for lane-changing.

- Obeying traffic rules

We introduce a probability $\left(p_{3}\right)$ to describe if the driver obey the evaluating traffic rule. That decided whether the driver would act as the traffic rule asked.

- Deviation of distance

When driving a car, $d_{1}$, presented in FIGURE II, is judged from drivers' experiences and could have some deviation. We can see that it will obey normal distribution. So, if we recorded them as two random variables $D_{1}$ we have:

$$
D_{1} \sim N\left(d_{1}, \sigma_{1}^{2}\right)
$$

Then, when a driver wants to change the lane, no matter what the real $d_{1}$ is, he has a chance of $p_{4}$ to decide to change lane, while $p_{4}$ is determined by the normal distribution:

$$
p_{4}=\int_{v_{2}}^{+\infty} \frac{1}{\sqrt{2 \pi} \sigma_{1}} e^{-\frac{\left(x-d_{1}\right)^{2}}{2 \sigma_{1}^{2}}}
$$

\section{The KeEP-Right-EXCEPT-TO-PASS Rule}

Take the Keep-Right-Except-to-Pass rule as an example, we evaluate it in some typical traffic conditions through our monetize model.

\section{A. Get the Parameters}

Assume that the highway is long enough to contain as cars many as possible at the same time; then chose a random section which can accommodate 200 cars (or cells) for one lane as the cellular space we study.

Following the idea of control variable method, we change only one parameter when analyzing a factor, keeping other parameters as we determined below.

$p_{1}$, the probability for randomization. Considering that it is a general rule which will be followed by all of the cells, it won't make any differences on comparison and analysis. So we set $p_{1}=0.3$ from beginning to end. Similarly, we have $p_{2}=0.2$.

When evaluating the Keep-Right rule, the main point need to specify is the meaning of $p_{3}$ (probability of obeying rules). $p_{3}$, here, gives the chance to determine if the car will go back to the former lane after changing one. And it will mainly take the two values:

- $\quad p_{3}=1$, the keep-right-rule is strictly observed

- $\quad p_{3}=0.5$, the rule perform practically no function.

For $p_{4}$, we have to determine $\sigma_{1}$ in advance. Assume that most of the distance determined by drivers could have a fluctuation in the range of $150 \% \sim 50 \%$ for the real distance, which is reasonable in reality. Then, we can estimate $\sigma_{1}$ from the " $3 \sigma$ " principle:

$$
\sigma_{1}=\frac{d_{1}}{6}
$$

$V_{\max }=9$ and $V_{\min }=6$. It is notable that we have unitized all of them and a unit equals to $5 \mathrm{~m} / \mathrm{s}$.

\section{B. Evaluate the Keep-Right Rule}

1) Compare with the Keep-Left rule

The driving direction will make some differences on drivers' estimation for distance in condition that the structure of vehicles is not changed. If the rule adjusts to keep vehicles in the left side with cabs also in the left, the deviation of distance judged by drivers will be expanded[9]. Thus, $\sigma_{1}$ is given by: (the distance varying in the range of $180 \% \sim 20 \%$ of $\left.d_{1}\right)$

$$
\sigma_{1}=\frac{4 d_{1}}{15}
$$

The results of monetizing is given in FIGURE III: 


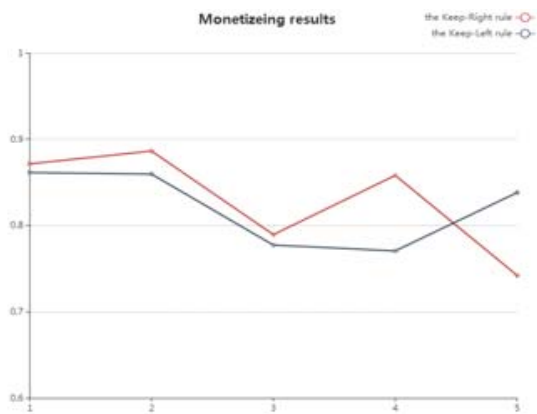

FIGURE III. MONETIZING RESULTS WITH THE KEEP-RIGHT OR -

\section{LEFT RULE}

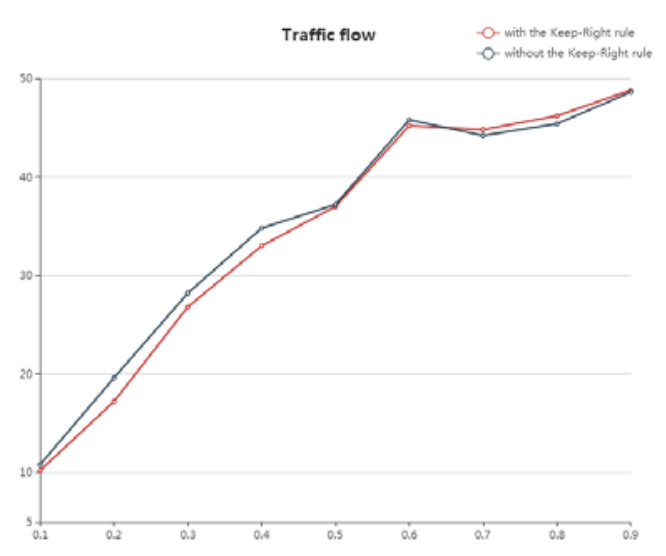

a)
It seems that the Keep-Right rule will perform better when other conditions keeps the same. But the hypothesis test tells us we can't conclude from the statistic results. That is to say, the two keeping rules are almost the same.

2) In light or heavy traffic

Whether the highway is in light or heavy traffic is presented by car-appearing coefficient $(\alpha)$.The following figures present the simulation results with car-appearing coefficient varying.

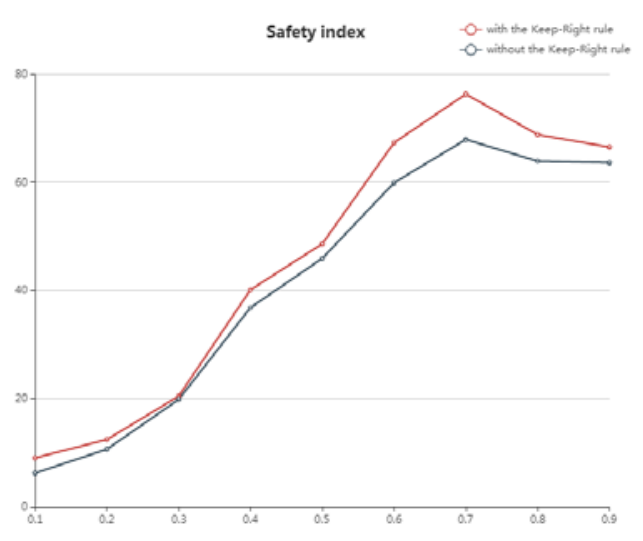

b)

FIGURE IV. CURVES OF THE TWO INDEXES WITH CAR-APPEARING COEFFICIENT VARYING: A) PRESENTS THE TRAFFIC FLOW, WHILE B)PRESENTS THE SAFETY INDEX

It is noticeable that when $\alpha$ is small, stick on the rule will have lower traffic flow, while whether there is a rule makes little difference in traffic flow in heavy traffic. And the characteristic of safety presents a different view. When $\alpha$ is small, the two curve is nearly coincided, while the curve for

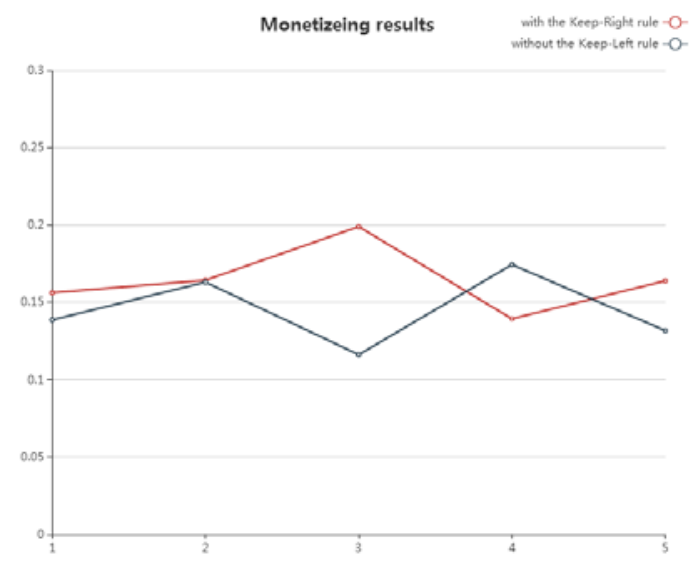

a) $p_{3}=1$ causing a higher value for the danger coefficient in heavy traffic.

The monetizing results of two extreme condition (light traffic when $\alpha=0.1$ or heavy traffic $\alpha=0.9$ ) is given in FIGURE.

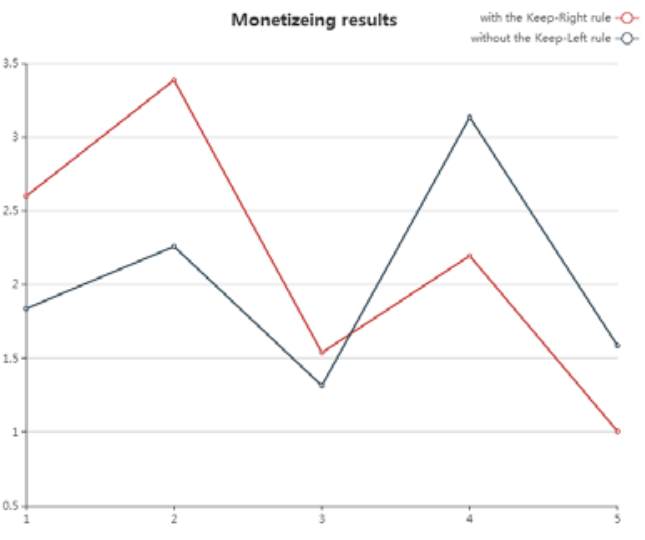

b)

FIGURE V. MONETIZING RESULTS IN DIFFERENT TRAFFIC CONDITION:A) FOR LIGHT TRAFFIC, WHILE B) FOR HEAVY TRAFFIC

The hypothesis test tells that we can conclude at a probability of $95 \%$ that the cost of road is higher with the keep-right rule, and that there are little differences with or without the keep-right rule in heavy traffic. 


\section{3) Different speed limitation}

Here we just adjust the maximum or minimum speed limitation to find out whether the speed limitations will make some difference, rather than find the "optimal" speed limitations. So, we adjust $V_{\max }=10$ and $V_{\min }=7$ separately.

We can conclude from the results (FIGURE) that overposted speed limit could promote traffic flow, but the keep-

Traffic flow
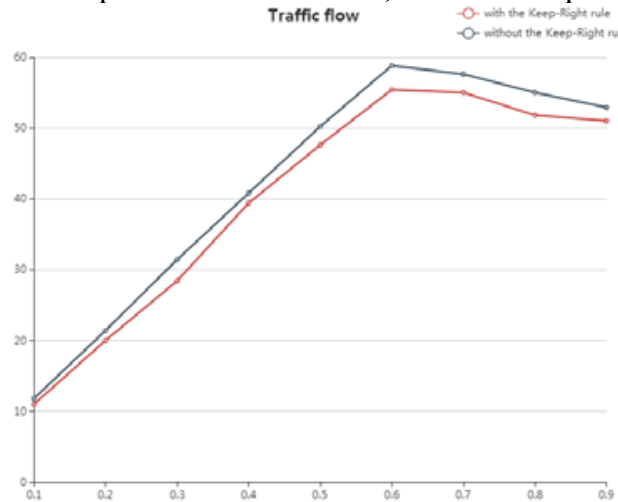

a)

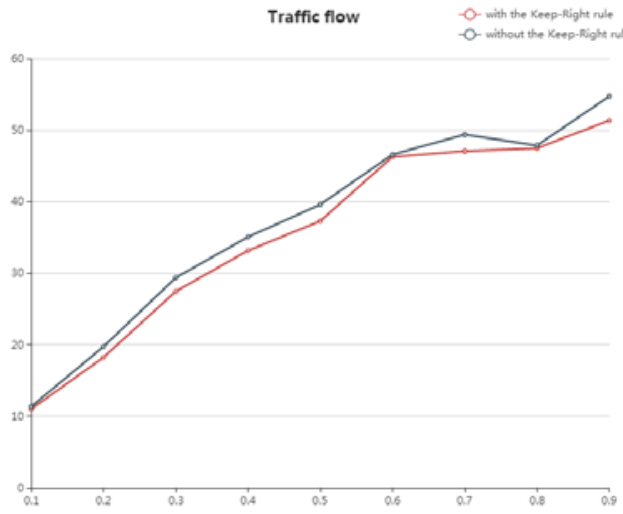

c)

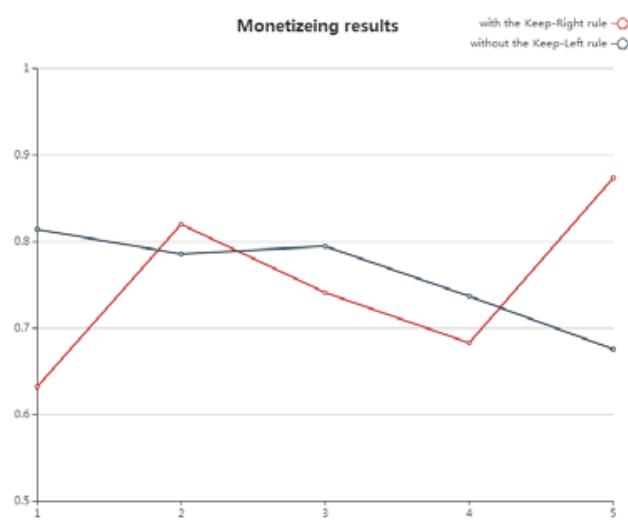

e) right rule will limit the promotion. The danger efficiency increased in all condition after rising speed limits. But we can still tell that it will be safer without the keep-right rule. And rising $V_{\min }$ is the most dangerous action with the same other conditions. And the hypothesis test tells that the cost is still higher with the Keep-Right rule when adjust speed limitation.

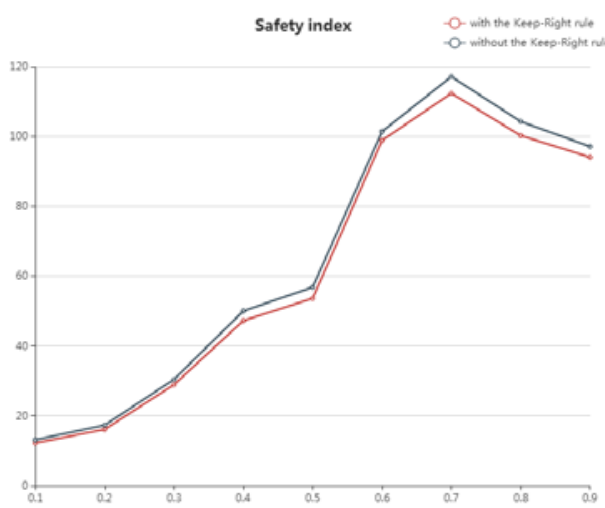

b)

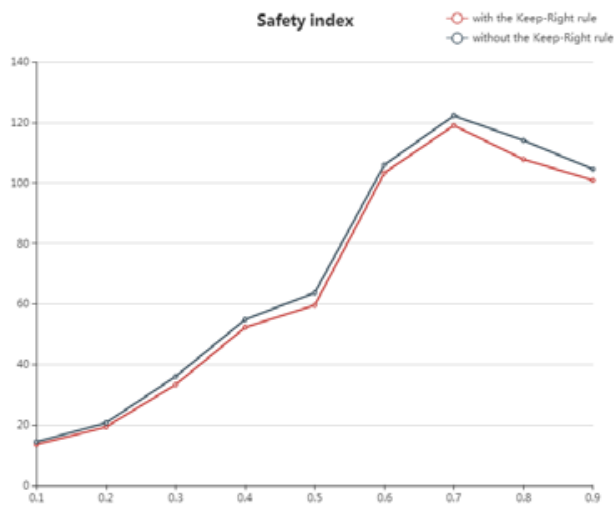

d)

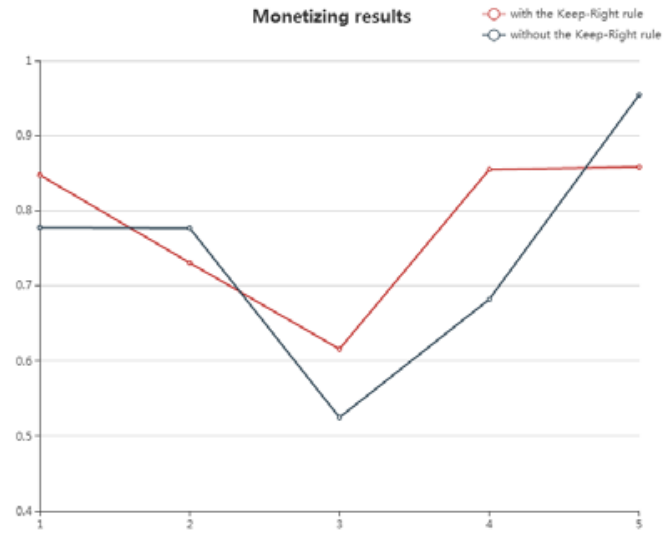

f)

FIGURE VI. EVALUATION RESULTS WHEN ADJUSTING SPEED LIMITATION. A) AND B) PRESENTS THE TWO INDEXES WHEN CHANGE THE MAXIMUM SPEED; C) AND D) PRESENTS INDEXES WHEN CHANGING THE MINIMUM SPEED; E) AND F) GIVES THE MONETIZING RESULTS, AMONG THEM, E) GIVES THAT OF CHANGING THE MAXIMUM, WHILE F) CHANGES THE MINIMUM. 
4) New rules from evaluation results

It seems that the Keep-Right rule doesn't perform well with our evaluation model. So, we need some adjustment for the rule. Analyzing the evaluation results in different conditions, we arise two main adjustments for the Keep-Right rule:

- Different speed limits on different lanes

According to the analysis for the different speed limit, increasing the speed limits can save the cost, and it will be apparently better if we have all of the lanes available rather than limit cars on a single lanes. But it will reduce the traffic flow if some vehicles could not achieve the posted speed. So,

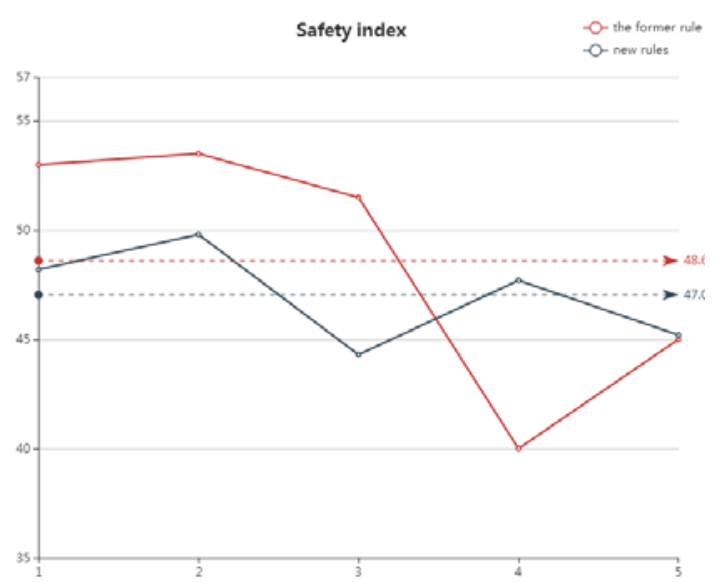

b)

a) we suggest the "Different speed limit in different lane" rule, that is to say, the speed limits in fast lane is higher than the slow lane, both on the maximum and the minimum.

- Loosen the keep-right rule strictly in holiday

Both the traffic flow and safety are to rise if we are not to obey the rule strictly. But it will also bring about some problems like finding it hard to overtake. Consequently, we propose that we can loosen that "keep-right" rule occasionally, such as in certain period like holidays.

Also evaluate the new rules with our model, the results (FIGURE VIII) tells that the new rules perform apparently better than the former one.

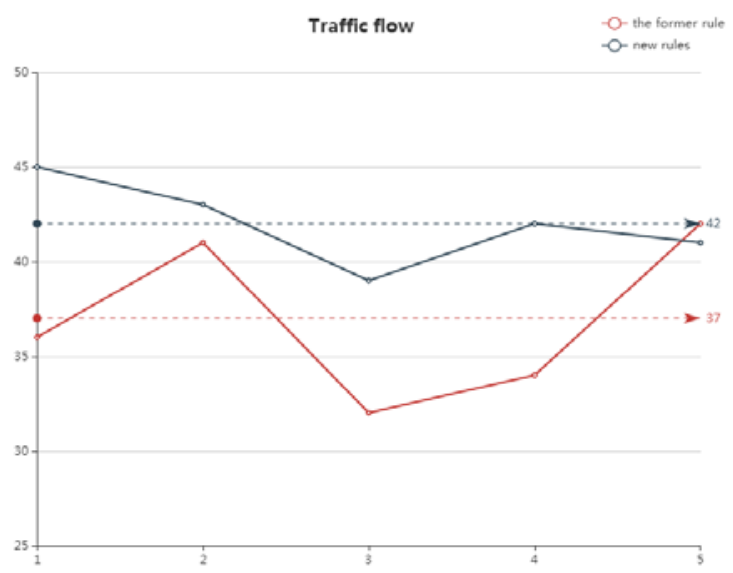

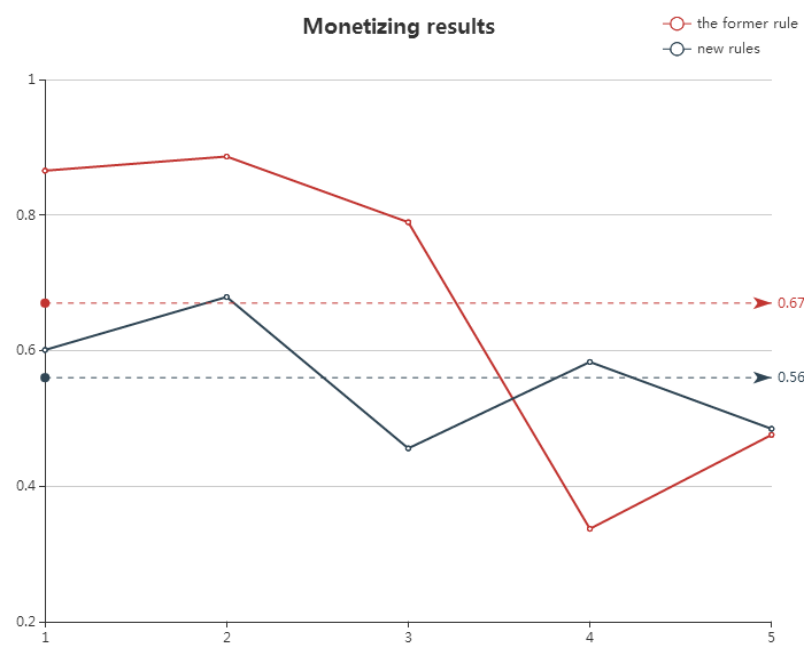

c)

FIGURE VII. EVALUATION RESULTS FOR THE NEW LAWS COMPARED WITH THE FORMER ONE: A) PRESENTS THE SAFETY INDEX, B) PRESENTS TRAFFIC FLOW, AND C) PRESENTS THE MONETIZING RESULTS

We know that "Different speed limits on different lanes" is actually a law for highway transportation in many countries. And that is powerful evidence that implies that our model could evaluate traffic laws objectively.

\section{CONCLUSIONS}

In this paper, we proposed a monetizing evaluation model for highway transportation. The main idea is to quantize the "cost" on the highway for cars, including the traffic flow and the security. And the transportation is modeled by cellular 
automation (CA), more specifically, based on the NaSch model.

Then, we take the Keep-Right rule as an example, evaluating it with our monetizing model and comparing with the Keep-Left rule. We also analyzed it under different circumstances: in heavy or light traffic, with different speed limitation.

Finally, we tried to propose some possible suggestion according to our evaluation results:

- $\quad$ Set different speed limits on different lanes;

- Loosen the keep-right rule strictly in holiday

We also evaluated the new rules with our evaluation model. The results suggested that the new rules perform better than the former one. And the results are also consistent with the virtual rules in many countries, which proved that our evaluate model is objective and effective.

\section{REFERENCES}

[1] Jiang Haifeng, Yang Fengyan,Han Wenyuan, Wang Lei.Study on driving behavior characteristics under the influence of traffic speed and warning signs[J].Public transportation technology, 2009(03)

[2] Treiber M., Hennecke A., Helbing D.. Deribation, properties, and simulation of a gas-kinetic based. Noulocal traffic model. Phys. Rev. E. 1999.59.239-253.

[3] Crener M., Ludwig J.. A fast simulation model for traffic flow on the basis of Boolean operations. J. Math. Comp. Simul. 1986. 28. 297-303.

[4] Zheng Rongsheng,Tan Huili,Kong lingjiang,Liu Muren.Research on two-lane multi-speed mixing traffic cellular automaton model[J].Journal of Physics, 2005, 54(8).

[5] Zhu Changsheng,Zhang Bo.Effect of Lane Changing Probability on Two $=$ dimensional Cellular Automaton Traffic Flow[J].Computer Engineering, 2012, 38(22).

[6] Wang Yongming,Zhou Leishan,Lv Yongbo.Cellular Automaton Traffic Flow Considering Flexibla Safe Space for Lane-changing[J].Journal of System Simulation, 2008, 20(5).

[7] Yan Jinan,Zhang Wei.Quantitative study of the stock market investment returns and risks of direct relationship[J].Journal of Tianjin University, 1999(4).

[8] LONG Gui-zhong,KONG Ling-jiang,LIU Mu-ren,ZHENG Rongsen .Study of NaSch Model's Segmentation[J].Journal of Guangxi Normal University(Natural Science Edition), 2008, 26(3).

[9] Wang Renguang,Bian Lijian,Liu Zhaodu,He Wei.Safety analysis of right-mounted steering wheel and right-side running vehicle design[J].Journal of Tianjin Institute of engineering demonstration, 2008, 18(3).

[10] Mark B., Mike M.. Car-following: a historical review, trans. Res. F. 1999. 2. 181-196.

[11] Bando M., Hasebe K., Nakayama A., et al.. Phys. Rev. E. 1995. 51. 1035.

[12] Helbing D., Tilch B.. Generalized Force Model of Traffic Dynamics. Phys. Rev. E. 1998.58. 133-138.

[13] Jiang R., Wu Q. S., Zhu Z.J.. Full velocity difference model for carfollowing theory. Phys. Rev. E. 2001.64.017101. 